11 a 13 de setembro de 2019 - Universidade de Brasília UnB

\title{
A GESTÃO DO CUSTO DO CICLO DE VIDA NO DESENVOLVIMENTO DE PRODUTOS DE DEFESA NAS FORÇAS ARMADAS BRASILEIRAS
}

Antonio Henrique Duarte (duarteantoniohenrique@gmail.com) - Universidade de Brasília Andrea Cristina dos Santos (andreakieck@gmail.com) - Universidade de Brasília

\section{RESUMO}

A Estratégia Nacional de Defesa (END), inicialmente lançada em 2008 e revista em 2012, estabeleceu a necessidade de reestruturação da capacidade operativa das Forças Armadas destacando a importância da indústria nacional de defesa ser a grande provedora desses equipamentos o que levou a substituição das importações por equipamentos desenvolvidos dentro do próprio país gerando um grande espiral de desenvolvimento conjunto entre a indústria de defesa e as Forças Armadas. Grandes avanços na área de desenvolvimento de produtos dentro das Forças Armadas foram alcançados, entretanto áreas principais como a gestão de custos ainda enfrenta grandes desafios. Por ser o método de Custo baseado no Ciclo de Vida o mais adotado pelas Forças Militares ao redor do mundo para desenvolverem seus produtos de defesa, este artigo tem por objetivo analisar o panorama atual de normas e metodologias que tratem de Gestão de Ciclo de Vida e métodos de Cálculo do Custo do Ciclo Vida utilizados pelas Forças Armadas Brasileiras, fazendo um paralelo com as boas práticas internacionais a fim de identificar lacunas e oportunidades de melhorias.

Palavras chave: Indústria de defesa; Custo do Ciclo de Vida; Gestão do Ciclo de Vida. 


\section{INTRODUÇÃO}

A Estratégia Nacional de Defesa (END), inicialmente lançada em 2008 e revista em 2012, estabeleceu a necessidade de reestruturação da capacidade operativa das Forças Armadas, destacando a importância da indústria nacional de defesa como a grande provedora desses equipamentos (BRASIL, 2012).

Deu-se então o início a um grande movimento de substituição das importações por equipamentos desenvolvidos dentro do próprio país, que somados a incentivos ficais para o setor, gerou um grande espiral de desenvolvimento conjunto entre a indústria de defesa e as Forças Armadas (BRASIL, 2017). Tal movimento levou a uma rápida necessidade de estruturação da gestão para fossem absorvidos todos os novos investimentos.

Desde então, grandes avanços foram feitos na área de desenvolvimento de produtos, as Forças Armadas passaram a adotar de maneira mais enfática o planejamento, execução, monitoramento e controle de suas grandes iniciativas de desenvolvimento por meio de Projetos e Programas Estratégicos (BRASIL, 2018).

Todavia, apesar de várias normas de gestão de projeto, programa e portfólio terem sido desenvolvidas ou adotadas pelas Forças para o desenvolvimento de produtos de defesa (BRASIL, 2018), ainda permanecem muitos desafios.

O desenvolvimento produto não é uma área nova, mesmo assim, um dos principais critérios que influenciam a tomada de decisão no processo de desenvolvimento de produto (PDP) continua sendo o custo. O sucesso do projeto de novos produtos é normalmente mensurado em termos do resultado financeiro (TYAGI, S., 2015).

Dentro da área de conhecimento custo, o método de Custo baseado no Ciclo de Vida de Produto tem sido o mais adotados pelas Forças Militares ao redor do mundo para desenvolverem seus produtos de defesa, uma vez que aborda de forma mais completa todos os custos envolvidos da vida de um produto e não apenas os custos de investimento inicial (WOODWARD, 1997).

Desta forma, o problema de pesquisa deste artigo é identificar o panorama atual de normas e metodologias que tratem de métodos de Cálculo do Custo do Ciclo Vida de Produto utilizados 
pelas Forças Armadas Brasileiras, fazendo um paralelo com as boas práticas internacionais a fim de identificar lacunas e oportunidades de melhorias.

Apesar de nosso objetivo ser o cálculo do Custo do Ciclo de Vida de Produto, não poderíamos analisá-lo sem antes identificarmos e analisarmos as metodologias de Gestão do Ciclo de Vida de Produto, uma vez que a existência do Ciclo de Vida de Produto é pré-condição para depois se possa calculá-lo.

\section{REVISÃO TEÓRICA}

A seguir foram descritos alguns conceitos básicos de custo, pois se fazem necessários para estudo do Custo do Ciclo de Vida de Produtos aplicado a projetos e programas.

Desembolso "é o ato do pagamento e que pode ocorrer em momento diferente do gasto.". O desembolso é um pagamento de um bem ou serviço não considerando quando ele for consumido e o mesmo ocorre na saída de recurso financeiro do caixa (BORNIA, 2010).

Por sua vez, gastos são os valores pagos pela organização por um produto ou serviço prestado. Se esse gasto foi pago no ato ele irá ter uma diminuição do ativo, e para pagar no futuro ele se tornará uma obrigação a pagar. O gasto então pode ser qualquer ação que a empresa faça, podendo ser um investimento para compra de algum equipamento, como um gasto com desperdício que não gera lucro (DUTRA, 2003).

Já custos representam os gastos relativos a bens ou serviços utilizados na produção de outros bens ou serviços (BRUNI; FAMÁ, 2008). Portanto, estão associados aos produtos ou serviços produzidos pela entidade. Custo são os gastos diretamente ligados a prestação de serviços, e os mesmos podem ser classificados como fixo ou variável, direto ou indireto (BOMFIM; PASSARELLI, 2006).

Um outro conceito muito importante é o de investimento. Segundo esse conceito, investimentos são todos os bens e direitos registrados no ativo das empresas para baixa em função de venda, amortização, consumo, desaparecimento, perecimento ou desvalorização (SANTOS, 2005). O investimento também pode ser definido como um gasto de aquisição de ativos com a intenção de obter benefícios. Pode-se definir também que todo custo é um investimento, mas nem todo investimento é um custo (BERTI, 2002). 
Despesas são valores dos insumos consumidos para outras funções que não a fabricação direta do produto. No caso abordado despesas são os valores dos insumos que não estão diretamente ligados ao desenvolvimento dos serviços prestados (BORNIA, 2010). As despesas correspondem a bem ou serviço consumido direta ou indiretamente para a obtenção de receitas. Não estão associadas à produção de um produto ou serviço (BRUNI; FAMÁ, 2008).

Encargos sociais, telefone, internet, comissões de vendedores, salários de vendedores, propaganda e marketing, juros de desconto, taxas de cobrança, taxas de serviços, entre outros são exemplos de despesas, e que ainda podem ser classificadas como despesas administrativas e comerciais (BORNIA, 2010).

Os métodos de custeio objetivam identificar os gastos inerentes ao processo produtivo, acumulando-os de forma organizada aos produtos. Estes custos podem ser aplicados a diferentes objetos tais como: produtos, departamentos, atividades, processos, ordem de produção, ou outras formas que o gestor possa demonstrar interesse (ROBLES JR, 1992). Os métodos existentes são: Pleno, Absorção, Variável/Direto e Por Atividade.

O Custeio Pleno, ou integral, é aquele que se caracteriza pela apropriação de todos os custos e despesas aos objetos. O Custeio por Absorção objetiva a apropriação de todos os gastos decorrentes somente do processo produtivo aos produtos, identificando os fixos, variáveis, diretos e indiretos (BOMFIM; PASSARELLI, 2006). O Custeio Variável, ou direto, tem o propósito de alocar aos produtos somente os custos identificados como variáveis, normalmente compostos pela matéria-prima e a mão-de-obra direta, consumidos no processo produtivo.

O Custeio Baseado em Atividades (ABC - Activity Basead Costing) procura identificar quais os custos das atividades e não dos produtos, pois defende a óptica de que os produtos consomem atividades e são estas atividades, e não os produtos, que consomem recursos(DUTRA, 2003).

No tocante aos métodos de Custeio do Ciclo de Vida, Nakagawa (1991) afirma que o sistema de custeio por ciclo de vida consiste na acumulação de custos por atividades ocorridas durante o ciclo de vida total de produto. Isto inclui não só custos repetitivos de produção, mas também aqueles que ocorrem apenas uma vez, tais como os que ocorrem durante a fase de desenvolvimento do produto e nas de suporte logístico (NAKAGAWA, 1991). Verifica-se, 
portanto, nesta colocação que o método sugerido é o "Activity Based Costing (ABC)". Robles (1992) também elege o "ABC" como o método compatível para custeio do ciclo de vida. Afirma, ainda, que a contabilidade por Atividades pode considerar na apuração dos custos das atividades a depreciação com base na vida tecnológica do equipamento ao invés dos valores apurados com base na vida útil (ROBLES JR, 1992).

O Custeio do Ciclo de Vida pode ser definido como "um método de calcular o custo total da propriedade durante toda a vida útil de um ativo". Nessa conceituação, além do custo inicial, todos os subsequentes custos esperados, significantes, assim como o valor residual e quaisquer outros benefícios quantificáveis a serem derivados (ELLRAM, 1994). Consequentemente, a técnica do Custeio do Ciclo de Vida é utilizada quando houver uma decisão sobre compra de um ativo que exigirá substanciais custos de operação e manutenção durante sua vida útil. Além disso, também afirma que, por exemplo, custos de operação e manutenção ao longo da vida de um edifício excedem em muito os custos iniciais, devendo, pois, serem considerados no processo decisório (ELLRAM, 1994).

Sob o enfoque de projetos e programas, vários autores têm discutido sobre como analisar os investimentos e considerando todos os custos envolvidos numa aquisição de bem ou serviço. Levando em consideração sua temporalidade, possuir um ciclo de vida bem definido, seu planejamento ser detalhado até o nível de tarefa e a complexidade de suas entregas, o Custeio baseado no Ciclo de Vida do Produto tem sido muito adotado (GUERREIRO; MEGLIORINI, 2003). Porém, depois de um projeto de investimento aprovado na empresa, geralmente os gestores de departamentos de compra ou afim, deparam-se com uma gama de modelos de produtos para aquisição, e que tradicionalmente são escolhidas as opções com menor custo ou os modelos das marcas mais reconhecidas do mercado, ou ainda, as que reduzirão o maior montante de mão-de-obra. Ocorre que tais decisões não levam em conta quais os impactos gerados por esta decisão no orçamento de anos futuros (GUERREIRO; MEGLIORINI, 2003), uma vez que o menor preço pode não ser a melhor decisão, pois podem estar embutidos nestes altos custos de manutenção, operação e descarte do bem (PMI, 2011).

O Custeio do Ciclo de Vida ou Custo do Ciclo de Vida não é uma coisa nova, foi originalmente projetado para fins de aquisição no Departamento de Defesa e ainda é usado mais comumente nas Forças Armadas ao redor do mundo (WHITE, 1976). Constitui-se de grandes 
itens a saber: (a) Custo de Aquisição, como pesquisa, desenho, teste, produção e construção; (b) Custo de Distribuição do produto, como transporte e manuseio; (c) Custo de Manutenção, como serviço ao cliente, manutenção no campo e na fábrica; (d) Custo de Operações, como instalações, energia, utilidades e impostos; (e) Custo de treinamento, de operadores e manutenção; (f) Custo dos Dados Técnicos; (g) Custo de Estoque, de peças, material e armazenagem; (h) Custos de aposentadoria e demissão de empregados (WILSON, 1983).

A soma de dos custos iniciais de aquisição e os custos que estão por vir, que serão incorridos na operação, manutenção e descarte do bem, o conjunto de todos estes custos é chamado de custo total do consumidor. O custo total de consumidor é uma ferramenta de suporte à tomada de decisão de aquisição de ativos que analisa os produtos de diferentes fornecedores para embasar uma seleção ou valoração aprimorada. $\mathrm{O}$ custo total do consumidor representa uma filosofia que tem como objetivo fornecer um entendimento do verdadeiro custo de fazer negócio com determinado fornecedor de um bem ou serviço em particular, pois apresenta informações que estão além do preço e incluem os custos relevantes que afetam criticamente a compra, demonstrando uma abordagem proativa (ELLRAM, 1994).

Também pode-se usar o método para as análises do tipo "fazer ou comprar" em projetos e programas, mesmo na decisão de comprar o bem ou serviço de um terceiro, é possível utilizálo para uma avaliação dos custos de terceirizar. Ocorre que o custo total do consumidor pode ser um método relativamente complexo para desenvolver um entendimento do custo real de uma aquisição devido a quantidade de variáveis envolvidas (ELLRAM, 1994). Entende-se, então, que a análise da aquisição dos ativos imobilizados dos Projetos e Programas Estratégicos do Exército podem ser realizadas com base no custeio do ciclo de vida do material, com base na informação do custo total do consumidor.

\section{METODOLOGIA}

Inicialmente realizou-se uma revisão teórica do assunto, através da pesquisa bibliográfica em livros e trabalhos científicos para embasamento teórico e num segundo momento foi feita uma pesquisa documental junto às Forças Armadas e Ministério da Defesa a fim de identificar e analisar quais Organizações Militares possuíam metodologias/doutrinas que tratassem da Gestão do Ciclo de Vida de Produto e Custeio baseado no Ciclo de Vida de Produto, assim como o nível de maturidade em que se encontram. 


\section{RESULTADOS E DISCUSSÃO}

Passa-se abaixo a análise das normas e legislações existentes no Ministério da Defesa do Brasil, Marinha do Brasil, Exército Brasileiro e Força Aérea Brasileira a respeito do assunto, assim como as de fontes internacionais notoriamente reconhecidas como modelos de boa prática.

\subsection{Exército Brasileiro}

A Força Terrestre possui as Instruções Gerais para a Gestão do Ciclo de Vida dos Sistemas e Materiais de Emprego Militar (EB10-IG-01.018). Esta norma foi aprovada em 18 de março de 2016 e tem como objetivo estabelecer uma sistemática para as atividades e os eventos que ocorrem durante o ciclo de vida dos SMEM (BRASIL, 2016). Segundo as Instruções Gerais para a Gestão do Ciclo de Vida dos Sistemas e Materiais de Emprego Militar do Exército, o processo de gestão do ciclo de vida dos SMEM é dividido em cinco fases: Fase de Formulação Conceitual, Fase de Obtenção, Fase de Produção, Utilização e Manutenção e Fase de Desativação (BRASIL, 2016). Apesar de prever as atividades envolvidas em cada fase, os responsáveis e o quando deve ser feito, as IG-01.018, especificamente na questão de custo não prever ou padroniza o como fazer. Ou seja, ela não possui a metodologia/doutrina de como realizar a precificação ou calcular o Custeio do Produto no Ciclo de Vida (BRASIL, 2016).

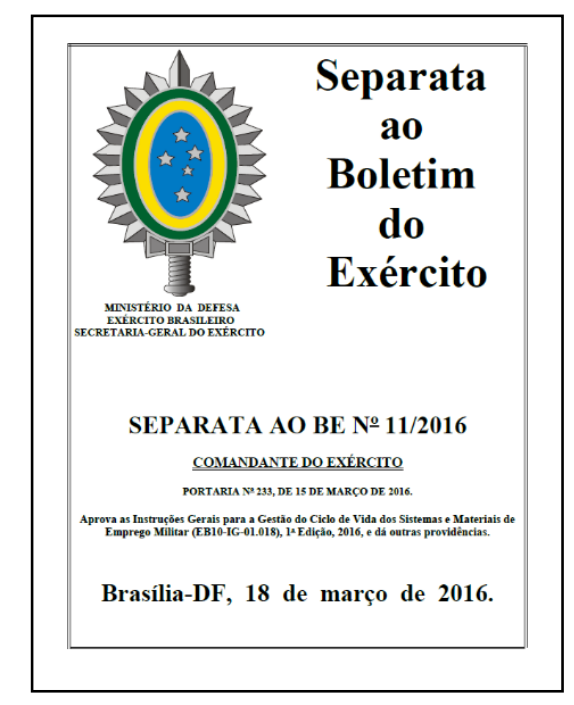

FIGURA 1 - Instruções Gerais para a Gestão do Ciclo de Vida dos Sistemas e Materiais de Emprego Militar do Exército (EB10-IG-01.018) 


\subsection{Força Aérea Brasileira}

A Aeronáutica possui a Diretriz que dispõe sobre o Ciclo de Vida de Sistemas e Materiais da Aeronáutica (DCA 400-6). Reeditada em 5 de março de 2007, a DCA 400-6 tem como finalidade ordenar o planejamento e a execução das fases e principais eventos do Ciclo de Vida, bem como regular tecnicamente a atuação, a interação e a responsabilidade dos Órgãos e Sistemas do COMAER que intervêm no processo (BRASIL, 2007). O Ciclo de Vida da Força Aérea é dividido em 09 (nove) fases: Concepção, Viabilidade, Definição, Desenvolvimento/Aquisição, Produção, Implantação, Utilização, Revitalização/Modernização ou Melhoria e Desativação (BRASIL, 2007). Segue abaixo a capa da norma:

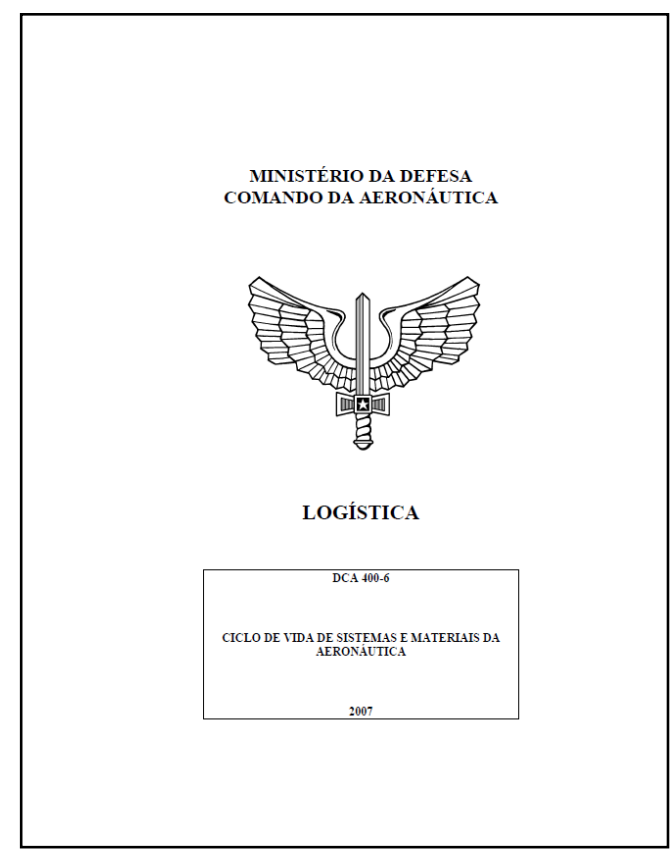

FIGURA 2 - o Ciclo de Vida de Sistemas e Materiais da Aeronáutica (DCA 400-6)

Apesar de prever as atividades envolvidas em cada fase, os responsáveis e o quando deve ser feito, a DCA 400-6, especificamente na questão de custo não prevê ou padroniza o como fazer. Assim como a IG do Exército Brasileiro, ela não possui a metodologia/doutrina de como realizar a precificação ou calcular o custeio do SMEM no ciclo de vida. 
A Força Aérea, porém, possui uma Norma Padrão de Ação a NPA/DFC/70 que padroniza a metodologia aplicada nas atividades de análise de custos da Subdivisão de Análise de Custos (SDANC) da Comissão Coordenadora do Programa Aeronave de Combate (BRASIL, 2014). A NPA/DFC/70 foi desenvolvida a partir do modelo existente no Ministério da Defesa Italiano e prevê todos os requisitos necessários a padronização de uma metodologia, como: definições conceituais, grupos de custos, modelos de tabelas de custos, etc (BRASIL, 2014). É uma metodologia para ser aplicada nas análises de custos das propostas apresentadas pelos fornecedores durante o processo de seleção/aquisição e permite a identificação da razoabilidade dos custos e preços ofertados, com base em documentos apresentados na proposta comercial e outras informações (BRASIL, 2014). Entretanto, suas definições, categorias de custos, modelos, etc, não cobrem todo o ciclo de vida, concentram se mais no que poderíamos chamar de investimento ou aquisição inicial.

Apesar de podermos adquirir juntamente com a aquisição um período de suporte logístico integrado (que cobriria os custos da operação), este suporte logístico integrado nunca irá cobrir todo o ciclo de vida. Outro ponto a ressaltar é que para essa metodologia funcione o fornecedor tem que está disposto a abrir a planilha de custos do sistema ou material. Tal premissa nem sempre irá se realizar, o que inviabiliza o método de quantificação. Desta forma, A NPA/DFC/70 se apresenta mais como uma excelente técnica de precificação, mas que não cobre todo o ciclo de vida e não pode ser empregada como única técnica de precificação que deve compor uma metodologia de custeio baseada no ciclo de vida. 


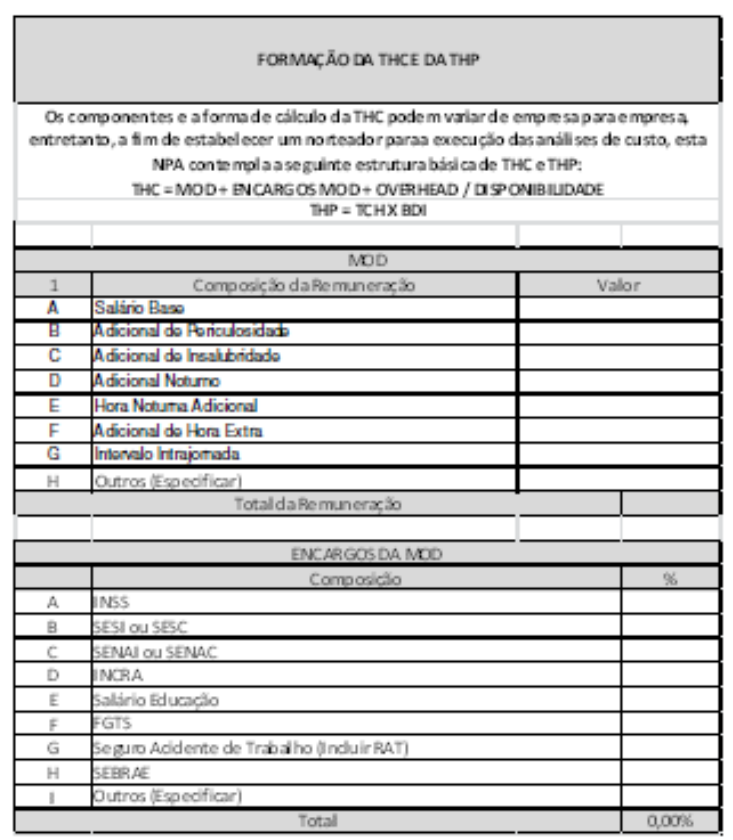

FIGURA 3 - Exemplo de um modelo de planilha prevista na NPA/DFC/70

(Força Aérea Brasileira)

\subsection{Marinha do Brasil}

Apesar da Marinha do Brasil empregar técnicas de custeio e ciclo de vida nos seus empreendimentos, ainda não existe na Força uma metodologia padronizando a elaboração dos mesmos. Segundo apurado, encontra-se em fase de elaboração.

\subsection{Ministério da Defesa (MD)}

Está em processo de aprovação no MD uma Doutrina de Gestão do Ciclo de Vida de Sistema de Defesa (SD), com a finalidade de padronizar a abordagem de gestão de ciclo de vida de SD e método para a estimativa e análise do custo do ciclo de vida, a serem executados pelas Forças Singulares e pelos órgãos subordinados ao MD. O ciclo de vida que será estabelecido pelo MD é composto por seis fases: concepção; desenvolvimento; produção; operação; apoio; e desfazimento conforme descrito abaixo: 


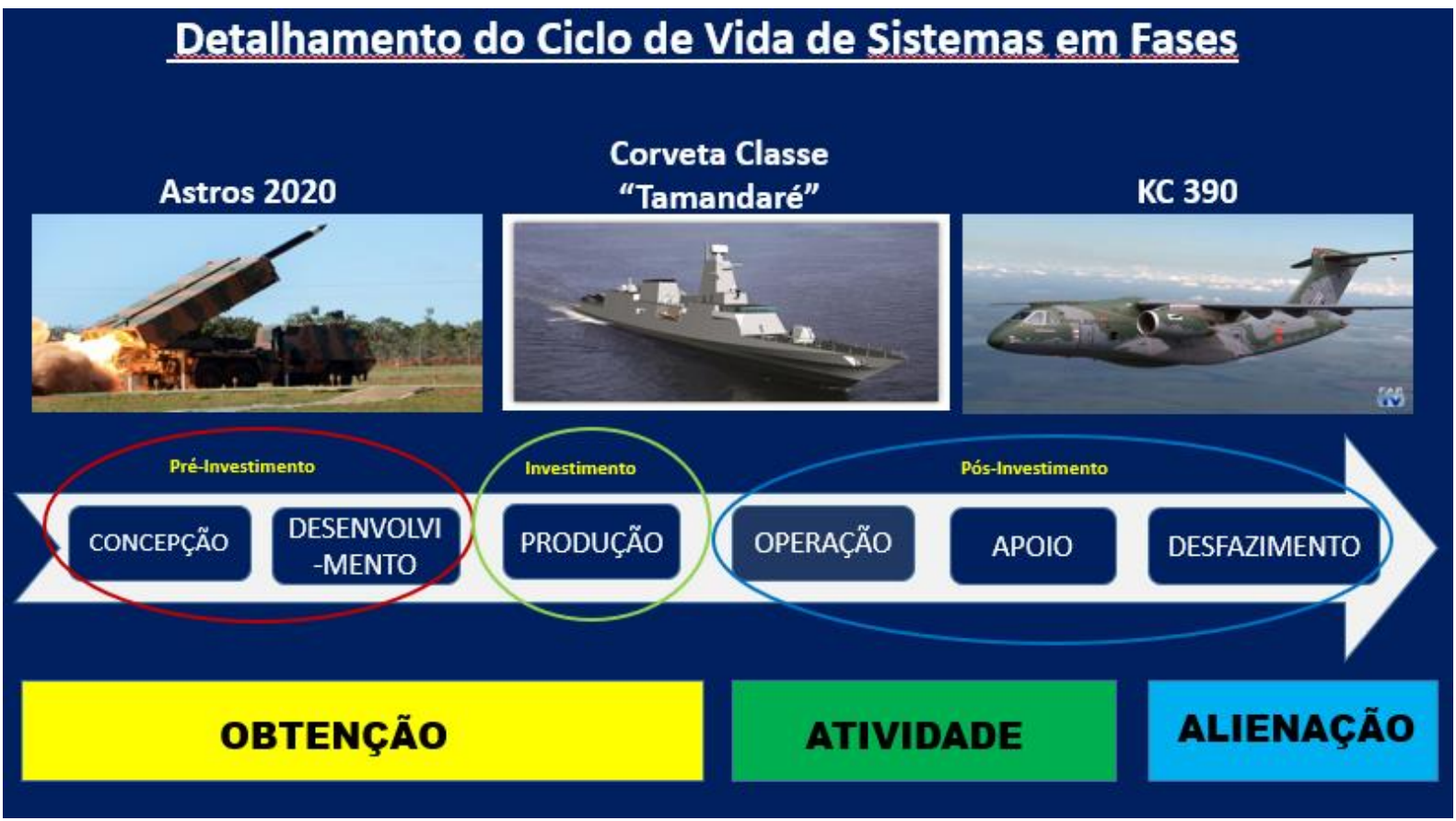

FIGURA 4 - Ciclo de Vida em processo de aprovação pelo Ministério da Defesa do Brasil

A metodologia de Custeio de Ciclo de Vida em elaboração pelo MD é descritiva, prevendo a elaboração de estimativas e análises de custo para o ciclo de vida de Sistemas de Defesa, sendo ainda aderente à bibliografia técnica especializada e as boas práticas observadas nas normas da Organização do Tratado do Atlântico Norte (OTAN). O CCV consiste em todos os custos diretos mais os custos variáveis indiretos associados à aquisição, operação e apoio e desfazimento de um novo equipamento ou capacidade, e é empregado para a análise de alternativas e para análises econômicas.

\subsection{Organização do Tratado do Atlântico Norte (OTAN)}

A North Atlantic Treaty Organization (NATO) ou Organização do Tratado do Atlântico Norte (OTAN), possui uma Política de Gerenciamento do Ciclo de Vida e uma norma genérica a AAP-20, que fornece orientação e informações padronizadas e para o gerenciamento de programas que incluem soluções de material (NATO, 2015). Estas devem ser usadas em conjunto com AAP-48, que por sua vez define os processos de Gerenciamento do Ciclo de Vida do Sistema (NATO, 2013). Este Ciclo é formado pelas fase Pré-conceito, Conceito, Desenvolvimento, Produção, Utilização (concomitante com o Suporte/Apoio) e Descarte ou Retirada. 


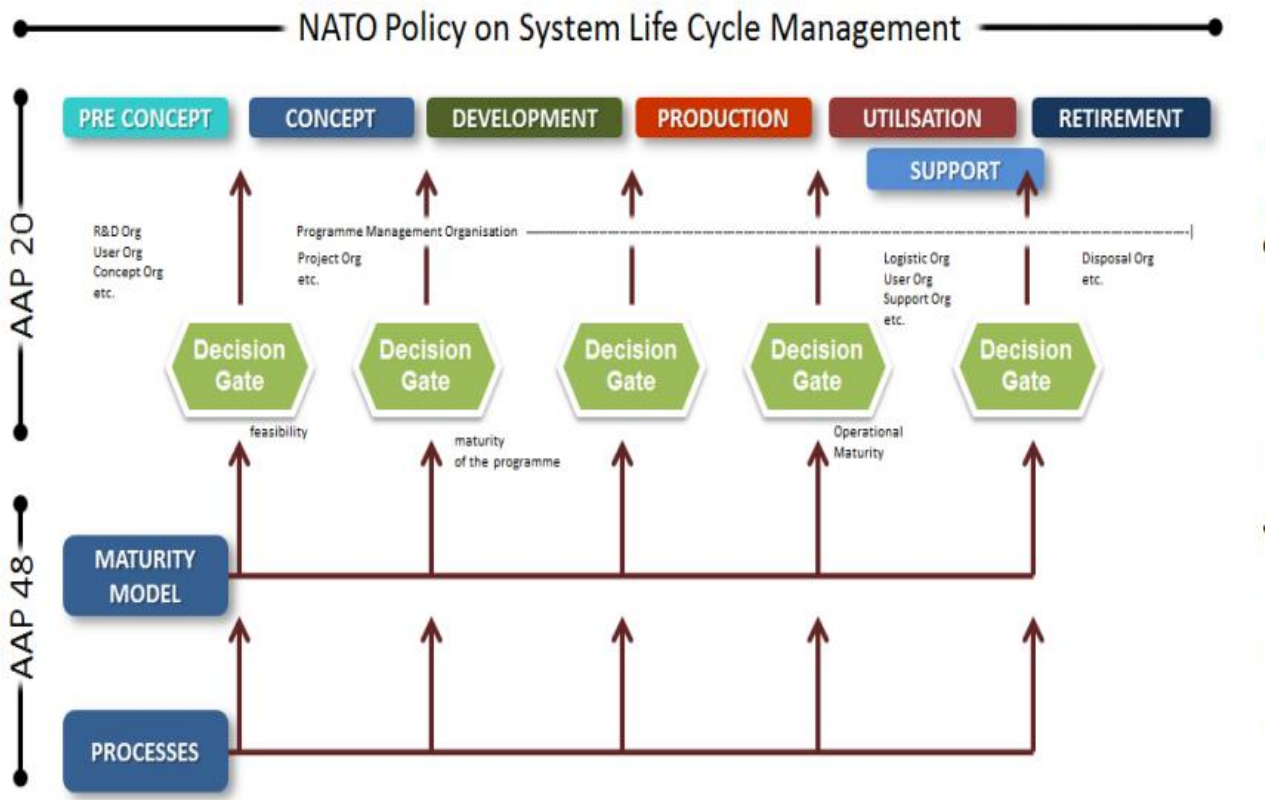

FIGURA 5 - Ciclo de Vida do North Atlantic Treaty Organization (NATO)

Em relação ao Custeio do Ciclo de Vida a OTAN possui o System Analysis and Studies Panel (SAS 54) que após estudos e debates realizados sobre as metodologias utilizadas pelos principais países aliados e participantes da OTAN, promulgou as melhores práticas no custeio do Ciclo de Vida que podem ser adotadas em fases dos Sistema de Programas de Armamentos da OTAN (NATO, 2007). Segundo este estudo, no desenvolvimento de estimativas de custo de ciclo de vida, todas as nações têm modelos desenvolvidos internamente que são similares e baseados em uma estrutura de divisão de custos definida. Os dados para esses modelos são estimados por métodos empíricos ou fórmulas paramétricas (para completar, algumas vezes as duas técnicas são empregadas). É considerado inclusive uma boa prática o uso de mais de um modelo para produzir uma estimativa de custo do ciclo de vida (NATO, 2007).

As boas práticas dos países revelam que os resultados do Custeio do Ciclo de Vida, independentemente da fase do programa/projeto, contribuem para que os gerentes possam tomar as melhores decisões sobre as opções apresentadas a eles. Essas opções podem incluir avaliação de despesas futuras, comparação entre soluções alternativas, gestão de recursos existentes, orçamentos, determinação dos fatores de custo associados ao desempenho, 
Indicadores ou requisitos do usuário principal, opções de aquisição e avaliação de oportunidades de redução de custos (NATO, 2007).

\section{CONCLUSÃO}

A pesquisa mostrou que no Brasil os estudos e normas vigentes ainda são incompletos (em relação a Gestão do Ciclo de Vida de Produtos) e inexistente em relação a Custo do Ciclo de Vida de Produtos. Mostrou também que já existem modelos externos bem desenvolvidos, completos e bastantes amadurecidos.

Podemos dizer que existem muitos métodos e modelos disponíveis para conduzir o Custeio Baseado no Ciclo de Vida. É importante entender a aplicabilidade e os limites de cada método e modelo em questão para adaptar adequadamente um modelo que se atenda às especificidades e peculiaridades das Forças. O Custeio Baseado no Ciclo de Vida é sem dúvida o mais completo modelo de custos para programas e projetos por gerar informações sobre todas as fases do programa/projeto com aplicação em vários momentos na gestão. Portanto é impositivo que sejam feitos esforços, prioritariamente em conjunto, para que o Ministério da Defesa assim como as demais Forças, desenvolvam normas e metodologias que padronizem tanto a Gestão do Ciclo de Vida e Custo do Ciclo de Vida de Produtos.

\section{REFERÊNCIAS}

BERTI, A. Custos: uma estratégia de gestão. São Paulo.: Ícone, 2002.

BOMFIM, E. DE A.; PASSARELLI, J. Custos e formação de preços. 4. ed. São Paulo.: Thomson, 2006.

BORNIA, A. C. Análise Gerencial de Custos: Aplicação em empresas modernas. Porto Alegre: Bookman, 2010

BRASIL, E. Portaria n 233, de 15 março de 20116. Aprova as Instruções Gerais para a Gestão do Ciclo de Vida dos Sistemas e Materiais de Emprego Militar (EB10-IG-01.018).Brasília, 2016.

BRASIL, E. O Exército Brasileiro em Transformação. Revista do Escritório de Projetos do Exército, v. 1, p. 44, 2018.

BRASIL, F. A. DCA 400-6. CICLO DE VIDA DE SISTEMAS E MATERIAIS DA AERONÁUTICA. Ministério da Defesa Comando da Aeronáutica. Brasília, 2007.

BRASIL, F. A. NPA/DFC/70: Atribuições da Subdivisão de Análise de Custos. Brasília, 2014.

BRASIL, M. DA D. Política Nacional de Defesa (PND) e a Estratégia Nacional de Defesa (END). $2^{\circ}$ Edição ed. Brasília: Ministério da Defesa, 2012.

BRASIL, M. DA D. Projetos estratégicos. ASCOM ed. Brasília: [s.n.].

BRUNI, A. L.; FAMÁ, R. Gestão de custos e formação de preço: com aplicações na calculadora HP 12C e Excel. São Paulo.: Atlas, 2008.

DUTRA, R. G. Custos: uma abordagem prática. São Paulo.: Atlas, 2003. 
ELLRAM, L. M. A taxonomy of total cost of ownership models. Journal of Business Logistics; ABI/INFORM Global, v. 15, n. 1, p. 191, 1994.

GUERREIRO, R.; MEGLIORINI, E. A percepção dos gestores sobre fatores competitivos nas empresas produtoras de bens de capital sob encomendas: um estudo exploratório. In: $3^{\circ}$ Seminário USP de Contabilidade. Anais...São Paulo.: 2003

NAKAGAWA, M. Gestão Estratégica de Custos. São Paulo.: Atlas, 1991.

NATO, N. A. T. O. TR-SAS-054: Methods and Models for Life Cycle CostingNeuilly, 2007.

NATO, N. A. T. O. AAP-48: Nato System Life Cycle ProcessesNeuilly, 2013.

NATO, N. A. T. O. AAP-20: Programme Management Framewok (NATO Life Cycle Model)Neuilly, 2015.

PMI, P. M. I. Practice standard for project estimating. Atlanta: Project Management Institute, Inc., 2011.

ROBLES JR, A. Contribuição ao Estudo da Gestão e Mensuração de Custos da Qualidade, no Contexto da Gestão Estratégica de Custos, Tese de Doutoramento. FEAUSP, 1992.

SANTOS, J. J. DOS. Análise de custos: remodelado com ênfase para sistema de custeio marginal, relatórios e estudos de casos. 4. ed. São Paulo.: Atlas, 2005.

TYAGI, S., ET AL. Value stream mapping to reduce the lead-time of a product development process. International Journal of Production Economics, p. 160, 2015.

WHITE, G. E. AND O. Life cycle costing”, Management Accounting. [s.l: s.n.].

WILSON, R. M. S. Cost Control Handbook. 2. ed. Londres: A Gower Handbook, 1983.

WOODWARD, D. Life cycle costing - theory, information acquisition and application. International Journal of Project Management, v. 15, p. 335, 1997. 